\title{
Mammary Neoplasms in Female Dogs: Identification of Cytopathological Criteria for Malignancy
}

\author{
Kamiguchi IE*, Moreira IM, Da Silva Trindade F, Zahn FS, Hataka A and Sousa Rocha N
}

Department of Pathology, Universidade Estadual Paulista Julio de Mesquita Filho, Botucatu, São Paulo 18618-970, Brazil

*Corresponding author: Emy Kamiguchi I, Department of Pathology, Universidade Estadual Paulista Julio de Mesquita Filho, Botucatu, São Paulo 18618-970, Brazil, Tel: 551236352302; E-mail: isabela.kam@hotmail.com

Received date: Oct 26, 2015; Accepted date: Dec 28, 2015; Published date: Jan 2, 2016

Copyright: (c) 2016 Kamiguchi IE, et al. This is an open-access article distributed under the terms of the Creative Commons Attribution License, which permits unrestricted use, distribution, and reproduction in any medium, provided the original author and source are credited.

\begin{abstract}
Mammary neoplasms have high incidence in both women and female dogs, with such a cytological resemblance between species that studies in canines might work as a baseline for research regarding the incidence in women, due to the relatively high incidence of this cancer, similar biologic behavior, large body size, comparable responses to cytotoxic agents, and shorter overall lifespan. Given the relevance of this neoplasm and the practicality of the cytological examination, studies listing the classification criteria for these tumors gains importance, aiding in diagnosis precision.
\end{abstract}

Aim and Objective: Therefore, this research paper aims at identifying and standardizing criteria that may be relevant for the diagnosis of malignancy in cytopathological preparations from mammary tumors in female canines. Once the cytopathological malignancy criteria are identified, we aim at verifying whether there is variation in the tumor's degree of aggressiveness considering the criteria established for mammary cancer in women.

Keywords: Mammary glands; Mammary neoplasms; Benignant tumors; Mesenchymal tissue; Metastatic cells

\section{Introduction}

Histologically speaking, the mammary gland is composed by parenchyma and stroma. The first is a secretory system composed by lobes organized in subunits, which, in turn, are composed by alveoli. These units are formed by a layer of epithelial cells with secretory capacity. Around these alveoli are myoepithelial cells, responsible for ejecting milk through hormonal response. The stroma is responsible for sustaining the parenchyma, being formed by connective tissue, fat, nerves and vessels $[1,2]$.

From birth until puberty, the breasts present the same growth rate than the rest of the body, but develops faster after puberty [1].

With the hormonal activity reached at the beginning of puberty, growth speeds up, especially on the parenchyma, due to the influence of estrogen and growth hormone (GH). The breasts remain largely unchanged. The final development happens in the case of pregnancy. In this case, the cells finish the differentiation process and attain functionality before the first conception due to the synchronous action of ovarian and pituitary hormones, including estrogen, progesterone, GW and prolactin, as well as placental lactogen. Before the end of the pregnancy, the breasts already produce a small quantity of milk. When the lactation period ends, there is an involution, in which part of the previously active alveoli degenerates and is substituted by adipose tissue [1].

Furthermore, the cytological classifications of the mammary neoplasms were divided according to criteria of tumoral grading - the Mouriquand and Guilford systems, which includes nuclear and architectural criteria - and nuclear criteria - the Black modified by Fisher, simplified Black system and Hunt system [3].

\section{Epidemiology}

Mammary tumors are the most common among female dogs, with $40 \%$ to $50 \%$ of those being malignant. There is a great resemblance between tumors afflicting female dogs and women. Although very common in humans, such tumors occur up to three times more in canines $[4,5]$. It is still unknown whether the animal's breed is a factor in the incidence of the disease [6].

About $60 \%$ of the lumps are located in the caudal abdominal and inguinal pairs and this high incidence is possibly related to the larger volume of adipose tissue in these areas [5,7].

According to studies, older animals present higher chances of developing malignant tumors, which are also larger, as opposed to younger animals, which generally present benignant and smaller tumors [5].

Several factors influence the onset of the disease, with hormonal factors being the most important one. Female dogs castrated before the first mating season present small chances of developing mammary neoplasms (around $0.5 \%$ ) considering that their contact with gonadal hormones was brief and that there was no significant development of the mammary glands until the castration, therefore avoiding future complications. Other authors state that the chances of developing tumors drop to $0.05 \%[5,7]$. When the animals are castrated after the first mating season, the risk increases to $8 \%$, while in animals castrated after the second or subsequent mating seasons the risk increases to $26 \%[5,8,9]$.

The administration of hormones during the animal's life may present a negative influence [5]. Progesterone used as a contraceptive seems to lead to a higher incidence of benignant tumors according to some authors [6]. However, the relationship between progesterone and the malignancy of mammary tumors is still being discussed [8], despite some authors defending the relationship between the administration of 
progesterone and a higher incidence of malignant tumors [5]. Other factors that may be related to the onset of tumors are genetic predisposition and diet. Obese animals with diets rich in fats often present a higher risk for the development of tumors [10,11]. The onset of false pregnancies is related to the incidence of malignant tumors in canines [6].

Benignant and malignant neoplasms may be classified according to the origin of the cells. They are often epithelial in origin, though may seldom be of mesenchymal or mixed origin. Mixed neoplasms present epithelial and mesenchymal tissue, with the later also possibly presenting cartilage or bone tissue [12]. Mixed mammary tumors present changes in the expression of myoepithelial cells, with studies proving the involvement of these cells with this type of tumor [13].

The tumor's degree of malignancy is directly related to the degree of differentiation of its cells. Low differentiation and high divergence from the original structure means high aggressiveness, with the inverse also being true as happens with the mammary gland's natural hormonal response. The degree of malignancy may also be assessed according to the number of atypical mitoses [6].

Most malignant tumors are metastatic. Tumor cells previously fixed in the region of the primary tumor, after mutations, gain enough motility to leave the location and establish themselves in other sites. Metastatic cells are usually transported by the lymphatic circulation, reaching the lymph nodes along the way [8].

The angiogenesis contributes to the rate of tumor metastasis since the vascular network is also a route for the distribution of tumor cells and contributes to growth [7].

There are several examinations for the diagnosis of mammary tumors in women, including cytopathological tests. These tests, however, are seldom used in canines and the mammary gland is often removed without previous examination. A mastitis may be removed instead of the cancer, representing serious risks to the animal upon defining the most adequate therapy [7].

When reaching the diagnosis of primary mammary neoplasm is needed, one should seek methods associated with quickness, ease of use, low invasiveness, low cost and high precision. Selecting the technique is not enough for the examination to represent an important method for the assessment of mammary cancer and showing knowledge regarding the structure is an important factor $[2,14]$. The cytopathological test shows all the characteristics mentioned. Through a simple capillary puncture, we may often obtain material for the diagnosis $[2,15]$.

In the assessment of the cytopathological test, there are several factors that should be considered in order to classify the lesion regarding malignancy. Regarding the cellular pattern of the sample, we should observe: high cellularity, small groups of cells with low or no organization and nuclear and nucleolar alterations [15-17].

Surgical procedures are considered elective treatment for mammary tumors in female dogs. The structures to be removed and the type of surgical approach depend on the extensiveness of the lesion. Small, firm and well-differentiated tumors require removal and a safe margin. More extensive tumors, in different locations, require unilateral or bilateral removal $[5,8]$.

An adjuvant chemotherapy may be conducted in malignant tumors, especially in case of metastases. The preservation of the breast and the excision only of the node are conducted in women, especially due to aesthetical concerns, being followed by chemotherapy or radiotherapy $[5,7]$.

Age contributes to the rate of survival both in humans and canines given that the younger the patient, higher the chances of overcoming the disease and better the recovery. However, age is not a determinant factor in whether the tumor is more malignant or less malignant, with only a small influence over the severity of the disease [8]. However, some authors state that the older the animal, higher the chances of the tumor being malignant [5]. The histological differentiation and the degree of malignancy have direct influence over the prognosis [7].

\section{Materials and Methodology}

\section{Normative research regulations}

The research project Neoplasia de mama em cadela: Identificação de critérios citopatológicos de malignidade (Mammary neoplasm in female dogs: Identification of cytopathological criteria for malignancy) has been submitted to the Ethics Commission in the Use of Animals (CEUA, Comissão de Ética no Uso de Animais) under protocol No. $63 / 2014$, being later approved by the commission.

The study included 20 female dogs seen in the cytology outpatient unit in the UNESP Veterinary Hospital, Botucatu/SP, based on previous consent by owners who agreed with the terms upon requesting medical care for their animals in the unit. The owners received explanations regarding the proposed procedures, respecting the possibility that such procedures might be refused. The participation of these animals did not interfere in any way with the routine of the unit.

\section{Clinical assessment}

After receiving approval, we advanced to the phase of clinical assessment of the animals. We obtained information regarding the animal's clinical history, paying special attention to the reproductive aspect. We also requested basic information such as age, weight, breed, time of evolution for the tumors in question and mapped the lesions regarding number, size and location in the mammary chain (clinical TNM).

\section{Cytopathological technique and sample processing}

The collection of samples was conducted by capillary puncture with a fine needle. For all samples obtained, we used consumable $30 \times 7$ or $30 \times 8$ hypodermic needles and consumable $10 \mathrm{~mL}$ syringes. After the puncture, the material was laid to rest over microscope slides using 8 $\mathrm{mL}$ of air in the syringe for propulsion and expelling the material in the needle. For each animal, we collected at least three samples distributed between Romanowsky and Papanicolaou stains. For the Papanicolaou stains, the sample was immediately submerged in $98 \%$ alcohol while for the Romanowsky stains the samples were air-dried and later fixated in methanol. After fixation, the stains were conducted according to their correct techniques. When ready, the slides were assembled with slides for reading and material preservation.

\section{Quality control and morphological criteria}

Regarding collection, all pieces obtained corresponded to the description and history of the respective animals, as well as with the animal's identification, which was correct and could be found in the system for data verification. All pieces provided enough material for 
cytopathological analysis, with none being discarded due to insufficient samples. A single sample was discarded due to not being a mammary tumor despite being considered as such initially. It was a mastocytoma and, therefore, was not included in the samples. All stains were conducted correctly, resulting in a material of good quality, and no sample resulted in poor stains which could not be assessed and classified.

The graphs below shows the criteria established for the cytopathological examination of mammary tumors in women, assembled for purposes of identification and morphological classification of samples.

\section{Determination of the malignancy criteria}

Initially, the samples with the Romanowsky stain were used to asses most characteristics and criteria presented, from cellularity to more specific criteria such as arrangement, microenvironment and cytoplasm, including quality control. Samples with the Papanicolaou stain had the role of complementing the aforementioned data, being used in the assessment of nucleus and nucleolus criteria, as well as in the differentiation of cell maturity.

\section{Method for analysis of the results}

The data noted in the work protocols were compiled, analyzed and plotted in graphics in order to determine the frequency and percentage of analyzed morphological criteria [18].

\section{Results}

Of the 20 canines, 10 were of no defined breed, two were Poodle, two were Daschound, one was Yorkshire, one was a German Shepherd, two were Pinscher, one was a Golden Retriever and one was a Rottweiler.

The average age was 10 years, with the youngest animal being 6years-old and the oldest animal 16-years-old. Two animals had this information missing in their file.

Regarding castration, only four animals were castrated, but none before the first mating season. Of the 20 animals, only two received contraceptives and four did not have the information available. The remaining owners denied the use of contraceptives. Eight animals had a case of false pregnancy in the past, 11 did not and one did not have the information available.

Regarding the weight of the animals, the average was $15.5 \mathrm{~kg}$. One of the animals did not have this information available.

The tumor evolution period was, in average, one year, reaching two or more years in some animals.

Of the 20 animals, seven presented at least one lesion only in an inguinal mammary, two only in a caudal abdominal mammary and five in both. Only five animals presented lesions in the cranial abdominal, caudal thoracic and/or cranial thoracic mammaries, with the incidence being much lower in these. One animal did not have this information in the history. Most animals had only one node in the affected mammary. Only five animals had two or more nodes and one animal had one node and two masses. Dimensions varied considerably, some between 0.5 and $1.0 \mathrm{~cm}$ in diameter and even one over $20 \mathrm{~cm}$ in diameter.
Regarding the cytopathological criteria, the predominant arrangement was papillary in $90 \%$ of the animals. The remaining $10 \%$ presented a cribiform arrangement (Figure 1).

All animals presented an epithelial component, 25\% presented a myoepithelial component and $70 \%$ presented high cellularity in the sample (Figure 2).

Regarding the cytoplasm, 95\% presented a low quantity of the component, which was irregular in at least $55 \%$ of the samples and basophilic in $85 \%$ of the cases.

Regarding nucleus and nucleolus, 75\% presented evident and multiple nucleolus, $45 \%$ presented multinucleation, $45 \%$ presented cariomegaly, 20\% presented cellular molding, 20\% presented micronucleus, $15 \%$ presented slotted nucleus and $100 \%$ presented gross chromatin (Figures 3 and 4).

Regarding the microenvironment, $75 \%$ presented neutrophils in the samples, $45 \%$ presented bone matrix, $20 \%$ presented cholesterol crystals, $70 \%$ presented red cells, $45 \%$ presented basophilic secretion and $40 \%$ presented eosinophilic secretion.

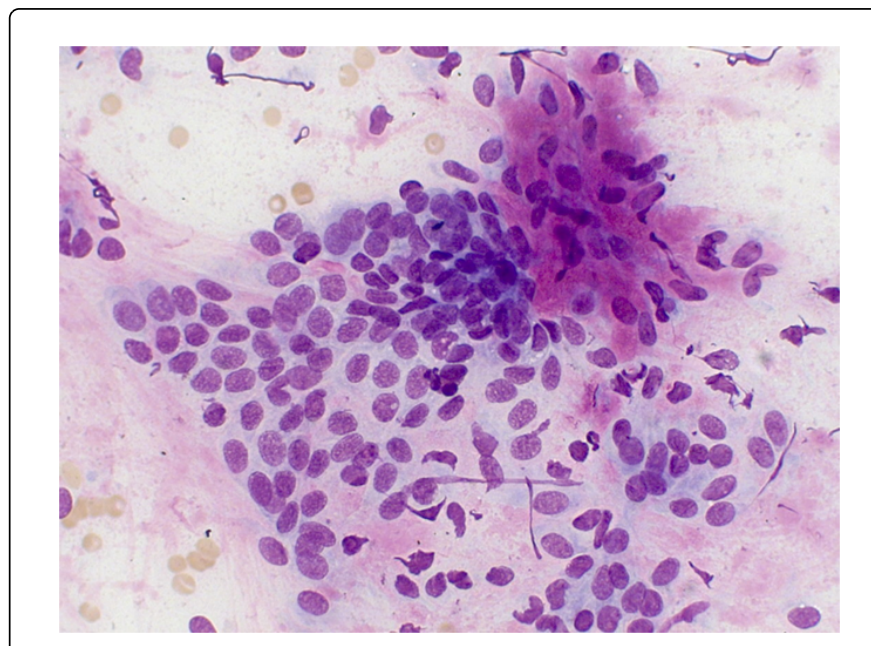

Figure 1: Components of a mixed mammary tumor in a female canine: epithelial cell (yellow arrow); myoepithelial cell (black arrow); bone matrix (red arrow).

\section{Discussion}

The age of the animals varied between 6 and 16 years old, with most animals (40\%) with ages between 10 and 13 years old, followed by animals between 6 and 8 years old (30\%). This creates a contrast with authors who reported a higher prevalence of animals between 12 and 13 years old [5], and others who reported a higher prevalence of animals between 9 and 11 years old [7], being closer to authors reporting ages between 7 and 12 years old [6]. Therefore, we may point towards the fact that these tumors are afflicting younger animals more frequently. Regarding to breed, given the common crossbreeding of animals in Brazil, it is hard to point that some breeds are more afflicted than others due to the fact that over $50 \%$ of the animals in the study are not of a defined breed. Regarding weight, although studies point towards a relationship between obesity and the development of tumors [11], given the heterogeneity of the samples and the presence of animals without a defined breed, we could not associate weight or obesity as an important factor for the affliction of these animals due to 
the fact that the body score or size of the breed are not present in the history.

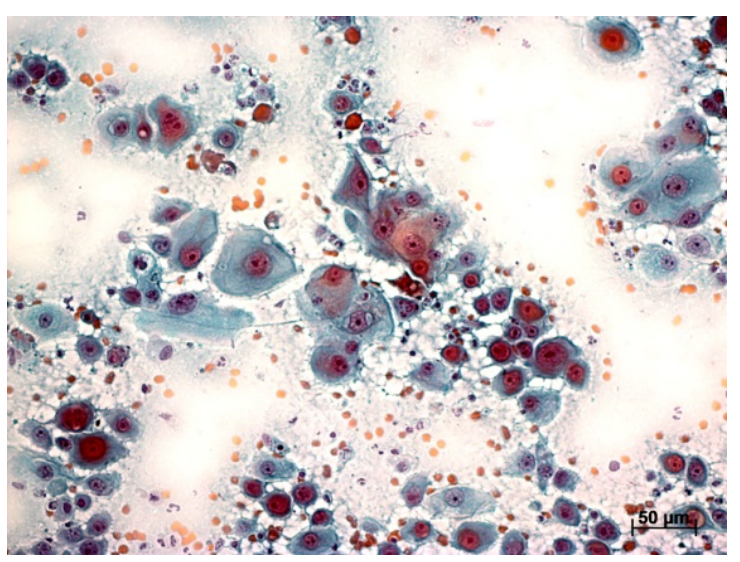

Figure 2: Nucleus and nucleolus criteria: cariomegaly (yellow arrow); anisocariosis (black arrow); anisocitosis (red arrow); multiple and evident nucleolus (green arrow).

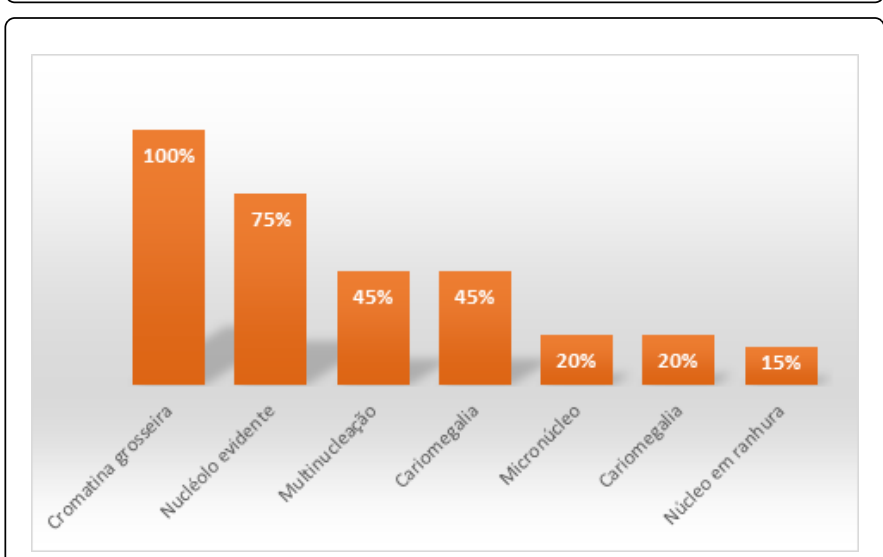

Figure 3: Frequency of nucleus and nucleolus criteria observed in the 20 cases of malignant mammary neoplasms in female canines.

It is important that, in the future, such information be present in the files for a better definition of this criterion. Regarding the reproductive history, none of the animals were castrated before the first mating season. Some were castrated, although later in life. This reinforces the statement by some authors that hormonal exposition during life increases predisposition to developing tumors and that early castration has shown good results when conducted before the first mating season in terms of reducing the risk of mammary tumors $[5,6,8]$. Regarding the occurrence of false pregnancies in a considerable amount of animals (40\%), despite the small sample size, indicates that such hormonal changes are related to this kind of neoplasm. This is in line with the conclusions of authors defending that animals with false pregnancies also present more malignant tumors $[6,8,9]$. Only $10 \%$ of the animals had a history of administration of hormones for contraceptive purposes, possibly indicating a low correlation with the development of tumors, a conclusion that is in line with some authors [6]. However, this study does not have enough data for such statement.

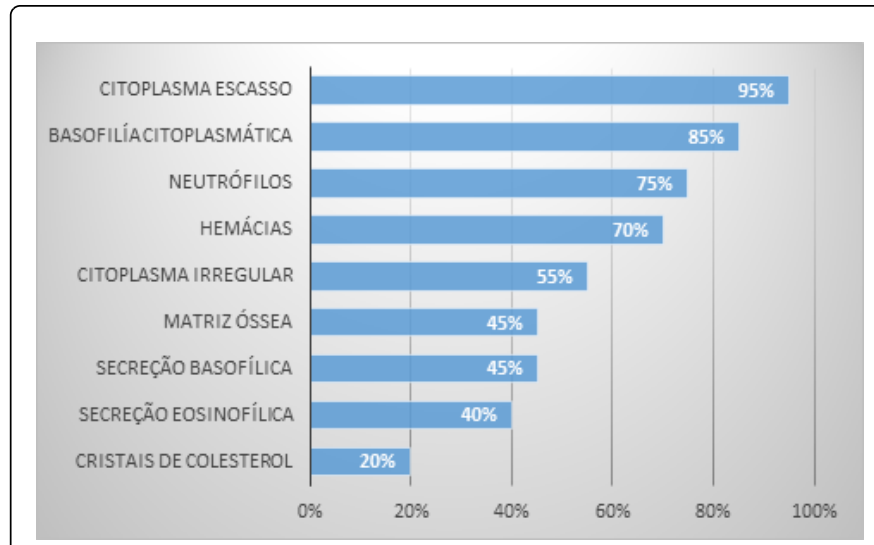

Figure 4: Frequency of criteria observed in the cytoplasm and microenvironment of the cytological samples obtained from 20 cases of mammary cancer in female canines.

Regarding location, the most afflicted mammary gland was the inguinal one, followed by the caudal abdominal one, which is in line with the literature $[5,6,8]$. Most animals presented single lesions (65\%), with only $35 \%$ presenting multiple lesions. This differs from the literature, which has a case selection with higher incidence of multiple lesions $[5,6,9]$.

Regarding the period of evolution, the average period was around a year, reaching two years or more in some cases. The owner's delay in seeking veterinary assistance for the animal is related to the development of malignancy and the worsening of the prognosis [5-7]. It is possible that some animals included in this study could have developed a lesser degree of malignancy if veterinary assistance had been sought earlier. It is important to raise awareness among owners regarding the risks of mammary tumors, how to prevent them and the importance of seeking veterinary assistance early, given that these are important factors impacting the patient's prognosis.

Regarding the criteria, the most common type of tumor found was papillary carcinoma (90\%), which is also the most commonly diagnosed in women nowadays [15]. Cellularity, considered an important malignancy criterion, was considered high in $70 \%$ of the samples. Other extremely relevant criteria are those regarding changes in the nucleus and nucleolus, including evident nucleoli. In this study, the nucleoli were evident and multiple in $75 \%$ of the samples, with the nuclear criterion being more commonly observed [19-21]. Still regarding these structures, criteria such as multinucleation, anisocariosis and cellular molding are also relevant towards the malignancy of tumors [15,22-24].

\section{Conclusion}

Based on the results observed in this study, we conclude that the cytopathological examination, when applied in female canines according to the established criteria in women, is considered an efficient auxiliary and/or alternative examination for the diagnosis of mammary tumors in female canines, presenting influence over the treatment choice for the animal and its prognosis. The criteria studied were important for the diagnosis of malignancy and for the classification in high or low degree of malignancy. 
Citation: Kamiguchi IE, Moreira IM, Da Silva TF, Zahn FS, Hataka A, et al. (2016) Mammary Neoplasms in Female Dogs: Identification of Cytopathological Criteria for Malignancy. J Cytol Histol 7: 392. doi:10.4172/2157-7099.1000392

Page 5 of 5

\section{References}

1. Neville MC (2001) Anatomy and physiology of lactation. Pediatric Clinics of North America 48: 13-34.

2. Dolka A (2014) The value of cytological examination of canine mammary tumours. Journal of Comparative Pathology 152: 65.

3. Danta NKA, da Cunha SG, Filho GO (2003) Grading Systems for Breast Carcinoma: Comparative Study of Cytohistological Agreement. Rev Bras Ginecol Obstet 25 Rio de Janeiro.

4. Shafiee R, Javanbakht J, Atyabi N, Kheradmand P, Kheradmand D, et al (2013) Diagnosis, classification and grading of canine mammary tumours as a model to study human breast cancer: a Clinico-Cytohistopathological study with environmental factors influencing public health and medicine. Cancer Cell International 13: 79.

5. $\quad$ Petrov EA, Ilievska K, Trojacanec P, Celeska I, Nikolovski G, et al. (2014) Canine mammary tumours - Clinical Survey. Macedonian Veterinary Review 37: 129-134.

6. Ribas CR (2012) Clinically relevant changes in dogs with mammary tumors staged. Archives of Veterinary Science 17: 60-68.

7. Sorenmo KU, Worley DR, Goldshmidt MH (2012) Tumors of the mammary gland. In: Withrow SJ, Vail DM, Page RL (Org.). Withrow \& MacEwen's small animal clinical oncology 5 (Edn). Saint Louis: Saunders 538-556.

8. Sorenmo K (2003) Canine mammary gland tumors. Veterinary Clinics of North America Small Animal Practice 33: 573-596.

9. Oliveira LO (2003) Epidemiological aspects of canine mammary neoplasia. Acta Scientiae Veterinarie 31: 105-110.

10. de Oliveira Brandão Y, Colodel MM, da Silva GN, Vexenat SC, Ferreira I, et al. (2013) Spontaneous mammary carcinomas in female dogs: association between the immunohistochemical degree of aggressiveness of tumors, intensity of DNA damage and residues of pyrethroids. Open Journal of Pathology 03: 133-137.

11. Lim HY, Im KS, Kim NH, Kim HW, Shinet JI, et al. (2015) Obesity, expression of adipocytokines, and macrophage infiltration in canine mammary tumours. The Veterinary Journal 203: 326-331.
12. Cassali GD, Bertagnolli AC, Ferreira E, Damasceno KA, de Oliveira Gamba C, et al. (2012) Canine mammary mixed tumor: a review. Veterinary Medicine Internacional 1-7.

13. Damasceno KA, Bertagnolli AC, Estrela-Lima A, Rabelo BS, Campos LC, et al. (2014) Versican expression in myoepithelial cells from carcinomas in caninemixed mammary tumors. The Veterinary Journal 200: 146-151.

14. Hodges J (2013) Using cytology to increase small animal practice revenue. Veterinary Clinics of North America: Small Animal Practice 43: 1385-1408.

15. Ministry of Health (2002) National Cancer Institute. Prevention Coordination and Monitoring - Comprev. Histopathology and cytopathology of breast lesions. Rio De Janeiro: Comprev pp: 7-13.

16. Raskin RE, Meyer DJ (2009) Canine and feline cytology: a color atlas and interpretation guide. 2nd (edn). Saint Louis: Saunders 472.

17. Colodel MM, Ferreira I, Figueiroa FC, Rocha SN (2012) Efficacy of fine needle aspiration in the diagnosis of spontaneous mammary tumors. Veterinary and Animal Science 19: 557-563.

18. Zar JH (1996) Biostatistical analysis: 3rd edition. New Jersey: Prentice Hall 718.

19. Howell L, Lin-Chang L (2005) Cytomorphology of Common Malignant Tumors of the Breast. Clinics in Laboratory Medicine 25: 733-760.

20. Stem Cell (2005) Restorer of life. Scientific American 39: 62-98.

21. Fletcher RH, Fletcher SW (2006) Clinical epidemiology: essentials, 4th edition. Porto Alegre: Artmed 288.

22. Harris JR (2009) Diseases of the breast: 4 th edititon. Philadelphia: LWW 1200.

23. Howard BA (2012) In the beginning: the establishment of the mammary gland during embryogenesis. Seminar in Cell and Developmental Biology 23: 547-582.

24. MacEwen EG (1990) Spontaneous tumors in dogs and cats: Models for the study of cancer biology and treatment. Cancer and Metastases Review 9: $125-136$. 